\title{
Agent-based Simulation Modeling to Measure the Effectiveness of UGV with Communication Repeater
}

\author{
[ Jaeyeong Lee, Sunwoo Shin, Chongman Kim ]
}

\begin{abstract}
Since the future warfare is getting more network centric rather than platform centric, its environment is getting more difficult and complex to estimate future system's operational effectiveness. Therefore it is challenging task to develop a methodology or approach to show the efficiency during a ground battle of the network centric warfare. In order to describe the reality of network environment, we are considering communication error effects depending upon terrain condition near each platform. The terrain condition is defined based on a small cell and its altitude in each cell. In this paper, we propose a simulation framework for how to measure the operational effectiveness of unmanned ground vehicle with considering communication repeater to compensate whenever communication error occurs in a ground battle scenario. The framework is processed with following three phases. At first, we consider all relational factors for input and output variables in communication network environment of all platforms. Secondly, build a simulation model and select a measure of effectiveness based on purpose of the system performance. Thirdly, execute a simulation model and produce MOE do the output analysis. We compared the results and showed how the effectiveness varies depending upon different scenario.
\end{abstract}

Keywords - Operational effectiveness, Modeling \& Simulation, Communication error, Communication repeater

\section{Introduction}

In previous war-game model, its output results were typically based on the Lanchester-type equations which have many unrealistic aspects to describe a complex war environment. Therefore, in these days, agent-based modeling $(\mathrm{ABM})$ draw attention because they can provide more realistic results according to their own decisions and actions for all platforms in a complex battle environment.

If the agent-based modeling method is applied to the wargame model, the fidelity of the model can be improved since many parameters and their interaction behaviors in the battlefield can be taken into account so that ABM makes wargame results more realistic. Hence agent-based modeling techniques would be more useful in the future simulation field.

Additionally, in previous war-game models, communication error effect (CEE) are not considered and their cause and effect results to weapon system effectiveness are not reflected either. However, $\mathrm{CEE}$ is the one of the most important factor in network centric warfare (NCW) because all platforms in a battle are connected each other not only to share target and damage information but also to order and report among related units based on chain of echelon. Therefore, in this study, we consider both agent-based modeling and communication error effects in a network centric warfare environment

\section{Basic concepts for modeling}

\section{A. Wargame Simulation Scope}

To build a new simulation framework to measure the UGV effectiveness, we setup a typical combat scenario in a small unit battleground which is different from theater level wargame. This means that our simulation scope narrows down to high resolution of the battlefield. The simulation framework we propose consists of three key themes such as ABM (Agent Based Modeling), CEE (Communication Error Effect), LOS (Line Of Sight).

For the representation of communication error effect, we depicted the altitude of the terrain in the model. For this purpose, different altitude level is expressed by each small cell area depending upon geographic surface pattern of the battle ground. When line of sight between two platforms is see through, no communication error effect would be applied. On the other hand, if line of sight between two platforms is blocked it will break into two cases of LOS descriptions. The Case 1 is called block cell LOS and Case 2 is called round cell LOS. The former assumed that levels of height are all the same in each cell and the latter assumed that levels of height are decreasing as it goes from the center of each cell of the terrain.

We use AnyLogic 7.0 to represent all these conditions and to validate the logic in war-game environment.

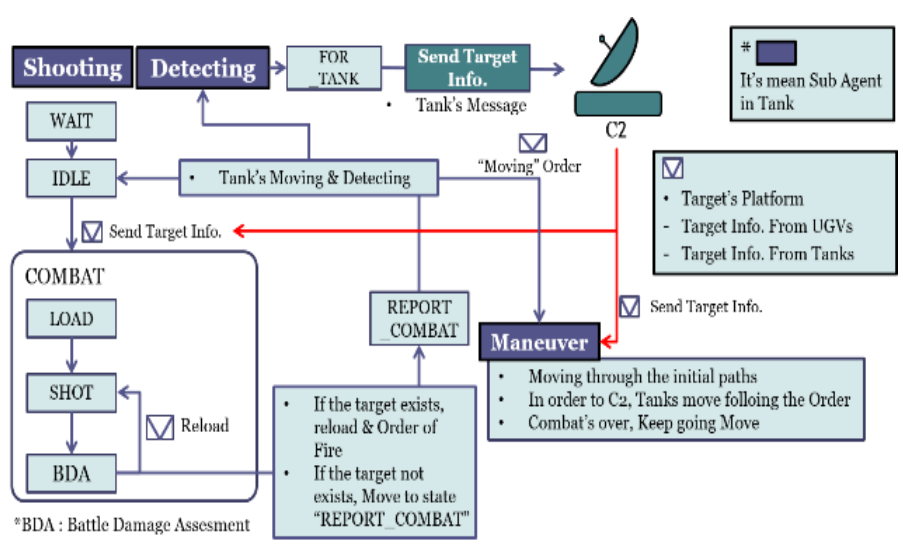

Figure 1. Overview of the Simulation Framework 


\section{B. Agent-based modeling}

In this paper, we study an agent-based simulation model framework to construct a scenario dependent war-game. In order to build war-game simulation using agent-based framework, rule-based approach must be established. For example, a war-game model requires a digital map called a battlefield agent, and many other types of agents acting on the battlefield such as Tank, APC, UGV, and C2, and each agent has its own acting rule-based framework.

\section{Communication loss function}

To show an effect of communication error within a wargame model, we use the path loss model, which is one of the functions describing communication in the physical layer between TX and RX, as a method of expressing communication. This model is based on free path loss function and is implemented by the communication channel environment and the distance between TX and RX

\section{Terrain Cells}

In the communication environment at battle field in the real world, there exists lots variable like communication terrain, power of communication, receive/transmission environment etc. for applying that communication environments in the real world, battle field in the model divides certain cells and that cells give variables that influence communication environments like altitude, terrain characters. So, in the Early model that applied cells describes square altitude (Figure 2. Case 1), but the latest model' s Cell have terraced altitude along the radius in cell (Figure 2. Case 2). In this way, if agents communicate between both, they have advantage more than case 1 so, in the latter case it gets realistic more than the former case.
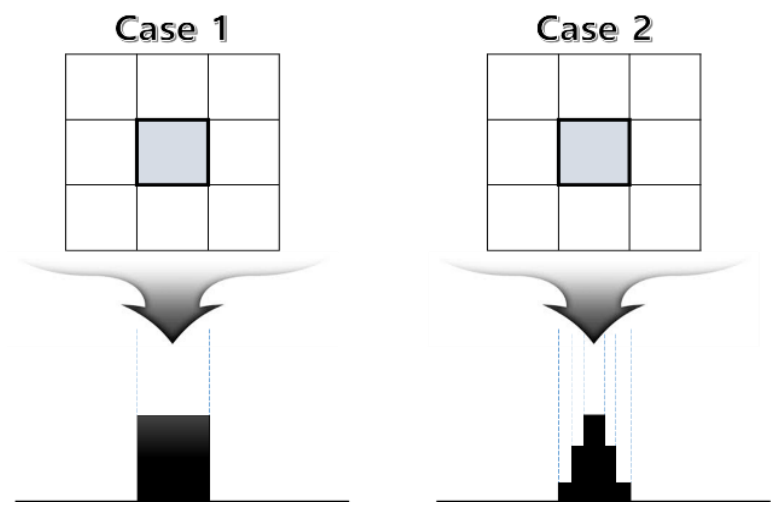

Figure 2. Two different scenario for terrain cells

\section{Model Development}

\section{A. Establishing agent structure}

There are three kinds of agents implementing to war-game. At first, a battlefield agent that implements battlefield: This battlefield agent has variables such as battlefield environment, cell-based terrain, altitude, topography type, etc., Secondly, a unit agent acting on the battlefield: a unit agent refers to all agents operating on the battlefield. For example, in this wargame model, the unit agent is represented by tank, UGV, and $\mathrm{C} 2$. Finally, the sub-agent that constitutes the unit agent: in the case of the sub-agent, it is an agent that is created to assist the function of the unit agent. For example, in the case of tanks, they have function-oriented sub-agents such as tank maneuvering, detection, and shooting. And all unit agents have framework that is automatically active on the battlefield agent.

\section{в. Communication implementation}

In order to represent for communication error in war-game model, it was implemented based on path-loss model and this is called a communication agent in the model. As shown in Figure 2, we consider 2 types of functions according to terrain environments, distance to TX to RX and availability of LOS.

As shown in Figure 3, there are several checkpoints (circled in red) which may be disconnected due to many reasons such as engagement damage, non-LOS, etc...

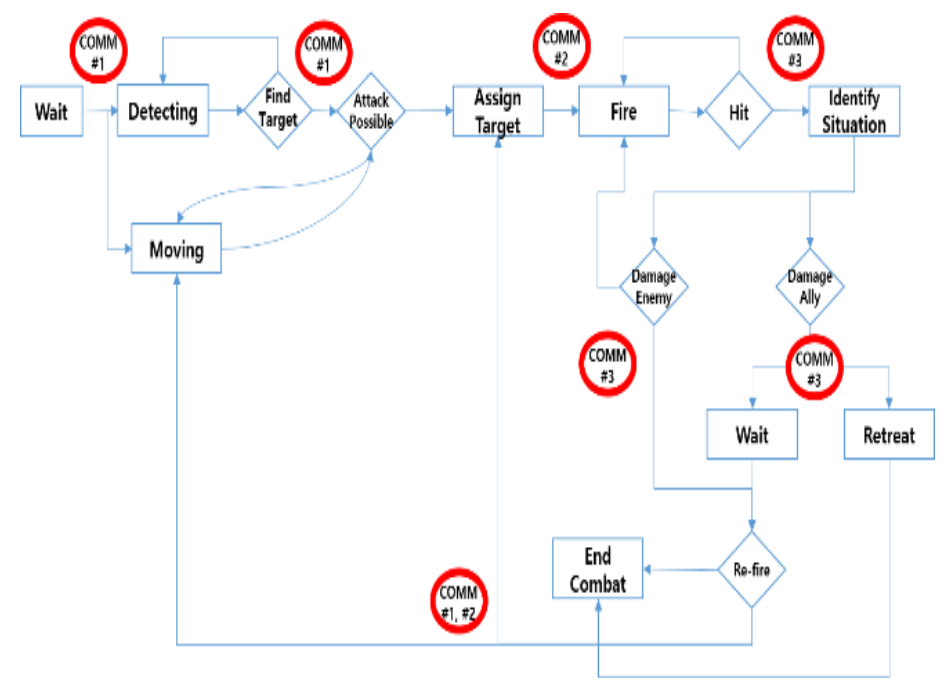

Figure 3. Checkpoints in the modeling process 


\section{Communication Repeater}

Communication repeater was used in order to establish backup system for the case of communication error in each checkpoint in Figure 3. Overall configuration for using communication repeater is depicted in Figure 4. Whenever a message from transmitter to receiver is not delivered properly in a certain checkpoint, a repeater is added to the checkpoint.

Modeling algorithm for communication repeater is shown in Figure 5. We also compare the results in case of having repeater to those in case of not having repeater.

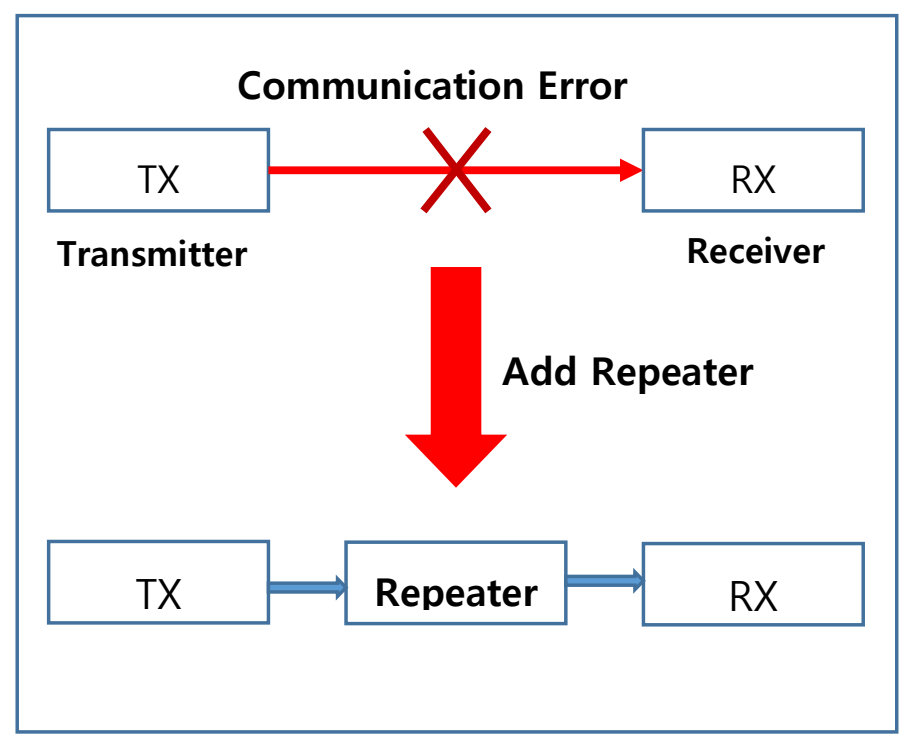

Figure 4. Usage of Communication Repeater

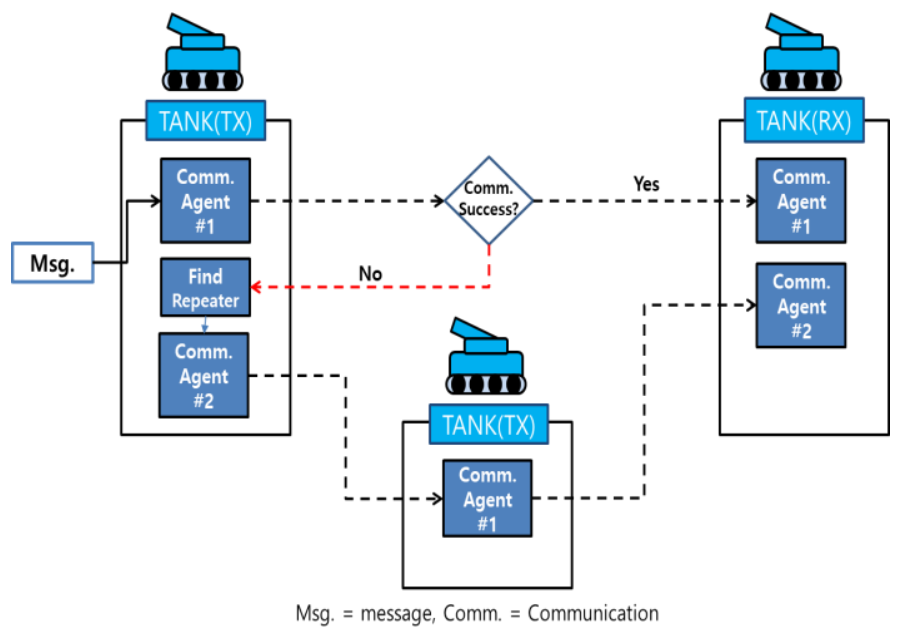

Figure 5. Modeling algorithm for Communication Repeater

\section{Measure of Effectiveness}

To analyze the effect of a simulation model, we need to determine a measure of effectiveness (MOE). The blue survival ratio (BSR) and red survival ratio (RSR) are calculated in the following processes.

1. Remaining assets (BT / RT) are calculated at the end of engagement for both sides.

2. Compare them to initial assets (B0 / R0) and count their ratio for both sides.

3. Calculate as in equation (1)

$$
B S R=\frac{B_{T}}{B_{0}} \times 100, R S R=\frac{R_{T}}{R_{0}} \times 100
$$

\section{Iv. Output Analysis}

We developed an agent-based simulation model with considering communication error effect in the model as a distinction from other research. To do that, we use a couple of communication path loss functions that consider different situations of terrain condition. The different terrain conditions we considered are either city or country which varies the level of LOS in communication among platforms. Figure 6 shows the results of varied MOE level depending upon each LOS condition respectively. The values in vertical line (y-axis) represent BSR in different LOS cases.

As we expected, as shown in Figure 6, BSR values are different from each LOS situation. The better LOS they have, the higher value of BSR they get after battle termination. This means that communication effect is highly depending upon the level of LOS. In other words, Clear LOS situation improves BSR sharply compared to Block Cell LOS situation, but slightly higher than Round Cell one.

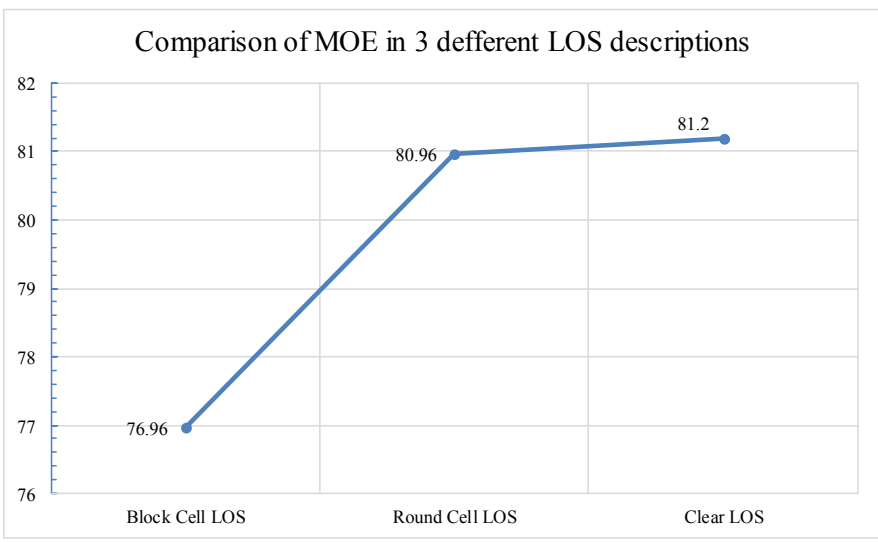

Figure 6. Simulation Results based on different LOS scenario 
We also get an analysis results from simulation when communication repeater has been applied to the checkpoint that fails to deliver the message via communication from a point to another.

Figure 7 shows differences among three cases as follows.

1. Only LOS : All terrain cell have LOS

2. LOS/NLOS : Some terrain cell have Non-LOS

3. Using repeater : Repeaters are added when needed

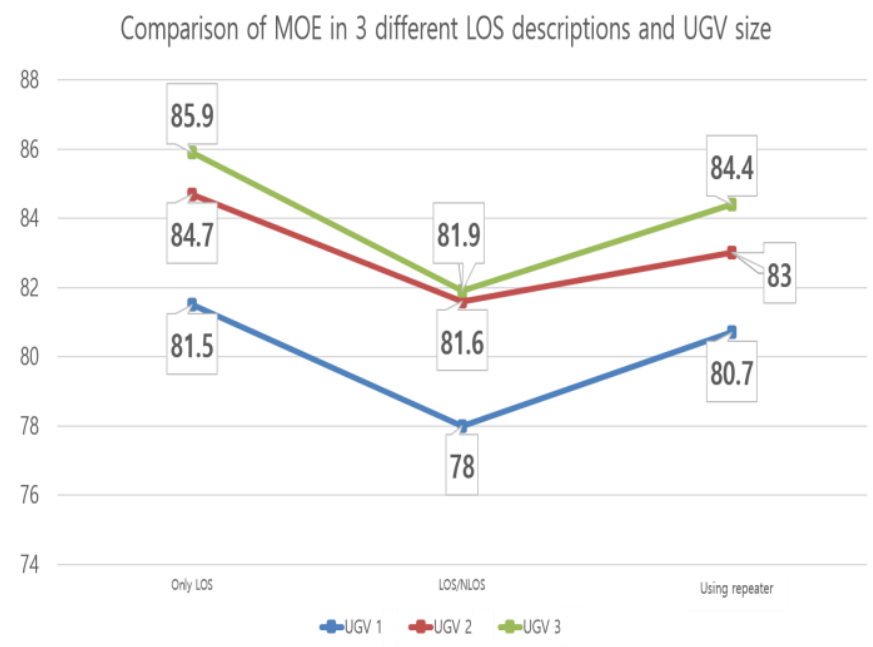

Figure 7. Simulation Results based on repeater

In Figure 7, we can see that about $5 \%$ of BSR is decreased when we consider Non-LOS from Only LOS situation, and $70 \sim 90 \%$ of reduction (from 1 to 2 ) is increased back when repeaters are added in each checkpoint having communication problem.

Figure 7 also shows how BSR increases depending upon the number of UGVs from 1 to 3 . Based on extra simulation results there is no big difference in the case of more than three UGV. Hence 2 3 UGV is the optimal number of assets in this scenario.

\section{Acknowledgment}

This study was supported by the Future Ground System Analysis Laboratory (UC130068ID) of the Agency for Defense Development in the Republic of Korea.

\section{References}

[1] G. Eason, B. Noble, and I. N. Sneddon, "On certain integrals of Lipschitz-Hankel type involving products of Bessel functions," Phil. Trans. Roy. Soc. London, vol. A247, pp. 529-551, April 1955. (references)

[2] J. Clerk Maxwell, A Treatise on Electricity and Magnetism, 3rd ed., vol. 2. Oxford: Clarendon, 1892, pp.68-73.

[3] I. S. Jacobs and C. P. Bean, "Fine particles, thin films and exchange anisotropy," in Magnetism, vol. III, G. T. Rado and H. Suhl, Eds. New York: Academic, 1963, pp. 271-350.

[4] K. Elissa, "Title of paper if known," unpublished.

[5] R. Nicole, "Title of paper with only first word capitalized," J. Name Stand. Abbrev., in press.

[6] Y. Yorozu, M. Hirano, K. Oka, and Y. Tagawa, "Electron spectroscopy studies on magneto-optical media and plastic substrate interface," IEEE Transl. J. Magn. Japan, vol. 2, pp. 740-741, August 1987 [Digests 9th Annual Conf. Magnetics Japan, p. 301, 1982].

[7] M. Young, The Technical Writer's Handbook. Mill Valley, CA: University Science, 1989. 